\title{
Switching State-Feedback LPV Control with Uncertain Scheduling Parameters
}

\author{
Tianyi He ${ }^{1}$, Ali Khudhair Al-Jiboory ${ }^{1}$, Sean Shan-Min Swei ${ }^{2}$, and Guoming G. Zhu ${ }^{1}$
}

\begin{abstract}
This paper presents a new method to design Robust Switching State-Feedback Gain-Scheduling (RSSFGS) controllers for Linear Parameter Varying (LPV) systems with uncertain scheduling parameters. The domain of scheduling parameters are divided into several overlapped subregions to undergo hysteresis switching among a family of simultaneously designed LPV controllers over the corresponding subregion with the guaranteed $\mathscr{H}_{\infty}$ performance. The synthesis conditions are given in terms of Parameterized Linear Matrix Inequalities that guarantee both stability and performance at each subregion and associated switching surfaces. The switching stability is ensured by descent parameter-dependent Lyapunov function on switching surfaces. By solving the optimization problem, RSSFGS controller can be obtained for each subregion. A numerical example is given to illustrate the effectiveness of the proposed approach over the non-switching controllers.
\end{abstract}

\section{INTRODUCTION}

The theory of Linear Parameter Varying (LPV) systems and gain-scheduling control techniques have witnessed significant attention from control community in the past two decades [1], [2], [3], [4], [5]. Many researchers applied this promising technique successfully in different engineering applications [6], [7], [8]. Since the analysis and synthesis problems of gain-scheduling control can be formulated as a convex optimization problem with Linear Matrix Inequality (LMI) constraints, this approach is very attractive for complex parameter-varying and nonlinear systems. Moreover, it offers an indisputable degree of computational and operational simplicity since the controller can be synthesized directly with guaranteed optimality.

However, it was found that controller design can be quite conservative when designing LPV controller over the entire parameter range and system performance is heavily sacrificed to obtain a feasible solution. In certain case, no feasible solution can be found for a given design problem. To reduce conservativeness and improve closed-loop system performance, references [9] and [10] present several switching LPV controller design methods. The idea of the switching LPV control is to divide the entire parameter region into subregions and then to design local LPV controller for each subregion to achieve optimal/sub-optimal system performance. The controllers are synthesized by formulating and solving Parameterized LMIs (PLMIs) using multiple

\footnotetext{
*This research is supported by NASA ARMD Convergent Aeronautics Solutions (CAS) Project.

${ }^{1} \mathrm{He}$, Al-Jiboory, and Zhu are with the department of Mechanical Engineering at Michigan State University. Emails: hetiany2 emsu .edu, aljiboor@egr.msu.edu, and zhug@egr.msu.edu.

${ }^{2}$ Swei is with the Intelligent Systems Division at NASA Ames Research Center. Emails: sean.s.swei@nasa.gov.
}

parameter-dependent Lyapunov functions [9] and certain switching strategy is needed to switch among these different LPV controllers according to current values of scheduling parameters. Furthermore, switching stability between any neighboring subregions is guaranteed via different switching strategies, such as hysteresis switching [11] or averagedwell-time switching [12]. Some applications of switching LPV controller design in practice have been demonstrated in [13], [14], [15].

However, the vast majority of gain scheduling design methods assume that scheduling parameters are accurately accessible in real-time for feedback control, i.e., scheduling parameters are perfectly measured or estimated, which is not realistic in practical applications. Since measurement errors in scheduling parameters could degrade system performance and even destabilize the closed-loop system, it is imperative to design robust gain-scheduling controllers with robustness against uncertainties in scheduling parameters. Although, this interesting control problem has been studied in literature before [16], [17], [18], to the best author's knowledge, this paper is the first one dealing with the measurement noises in switching LPV control framework.

The authors addressed the robust gain-scheduling control problem before in [8], [19]. In [8], PLMIs synthesis conditions were developed to synthesize state-feedback robust gain-scheduling controllers with the guaranteed closed-loop performance subject to additive uncertainties in scheduling parameters. This approach converts the controller synthesis problem into an optimization problem of PLMIs with line search that can be solved by efficient relaxation procedures [20], [21] and software tools [22]. However, the approach presented in [8] is not for the switching LPV control. Motivated by past results of LPV control with noisy parameters and the switching techniques, it is believed that better performance can be achieved using switching LPV control with noisy parameters, especially for the case with large parameter variation range. More precisely, based on the results in [8], this paper develops new PLMI conditions to design Robust Switching State-Feedback Gain-Scheduling (RSSFGS) controllers, where the entire parameter region is divided into multiple overlapped subregions and a family of RSSFGS controllers will be designed individually for each subregion. Hysteresis switching strategy with multiple parameter-dependent Lyapunov function are adopted to guarantee the switching stability between any neighboring controllers.

This paper is organized as follows: a brief review of LPV systems and problem formulation of RSSFGS control are 
given in Section II. PLMI synthesis conditions are derived in Section III. A numerical example is presented in Section IV to illustrate the effectiveness of proposed approach. Finally, conclusions are given in Section V.

\section{PROBLEM FORMULATION}

Consider the following affined LPV system:

$$
\begin{aligned}
& \dot{x}(t)=A(\theta(t)) x(t)+B_{u}(\theta(t)) u(t)+B_{w}(\theta(t)) w(t) \\
& z(t)=C(\theta(t)) x(t)+D_{u}(\theta(t)) u(t)+D_{w}(\theta(t)) w(t)
\end{aligned}
$$

where $x(t) \in \mathbf{R}^{n}$ denotes LPV open-loop system state; $u(t)$ denotes control input; $w(t)$ is the disturbance input; and $z(t)$ is the controlled output. The open-loop matrices have the following compatible dimensions $A(\theta) \in \mathbf{R}^{n \times n}, B_{u}(\theta) \in \mathbf{R}^{n \times m}$, $B_{w}(\theta) \in \mathbf{R}^{n \times r}, C(\theta) \in \mathbf{R}^{p \times n}, D_{u}(\theta) \in \mathbf{R}^{p \times m}, D_{w}(\theta) \in \mathbf{R}^{p \times r}$. The system matrices are assumed to be affine parameterdependent on scheduling parameter $\theta(t)$ such that any of the open-loop matrix in (1) can be expressed in terms of the scheduling parameter as,

$$
A(\theta(t))=A_{0}+\theta(t) A_{1} .
$$

Scheduling parameter $\theta(t)$ is assumed to be onedimensional, and it is affected by measurement noise in real-time. Thus, $\theta(t)$ represents actual scheduling parameter, $\delta(t)$ represents measurement noise, and $\tilde{\theta}(t)$ measured signal such that,

$$
\tilde{\theta}(t)=\theta(t)+\delta(t) .
$$

Scheduling parameter and measurement noise are both assumed to be bounded as,

$$
\begin{aligned}
& \underline{\theta} \leq \theta(t) \leq \bar{\theta}, \\
& \underline{\delta} \leq \delta(t) \leq \bar{\delta},
\end{aligned}
$$

also, the rates of changes of these parameters are assumed to be bounded as,

$$
\begin{aligned}
\underline{b}_{\theta} & \leq \dot{\theta}(t) \leq \bar{b}_{\theta}, \\
\underline{b}_{\delta} & \leq \dot{\delta}(t) \leq \bar{b}_{\delta},
\end{aligned}
$$

Consider the interval set for the measured scheduling parameter,

$$
\Theta:=\{\tilde{\boldsymbol{\theta}}(t) \in \mathbf{R}: \underline{\tilde{\theta}} \leq \tilde{\boldsymbol{\theta}}(t) \leq \overline{\tilde{\theta}}\} .
$$

The scheduling parameter set $\Theta$ is divided into finite numbers of subsets by a family of switching surfaces. Adjacent subsets are overlapped by each other to undergo hysteresis switching of controllers when scheduling parameters vary across switching surface.

In order to guarantee the stability in the switching region, the size of overlapped subset should be larger than that of parameter uncertainty, otherwise, parameter uncertainty cannot be able to be robustly handled by adjacent switched LPV controllers. Each subregion and overlapped subset of scheduling parameter are defined as follows,

$$
\begin{aligned}
& \Theta^{(j)}:=\left\{\tilde{\boldsymbol{\theta}}(t) \in \mathbf{R}: \tilde{\tilde{\theta}}^{(j)} \leq \tilde{\boldsymbol{\theta}}(t) \leq \overline{\tilde{\theta}}^{(j)}\right\}, j \in \mathbf{N}_{J}, \\
& \boldsymbol{\Theta}^{(j, j+1)}:=\left\{\tilde{\boldsymbol{\theta}}(t) \in \mathbf{R}: \overline{\tilde{\boldsymbol{\theta}}}^{(j)} \leq \tilde{\boldsymbol{\theta}}(t) \leq \underline{\tilde{\boldsymbol{\theta}}}^{(j+1)}\right\}, j \in \mathbf{N}_{J-1} .
\end{aligned}
$$

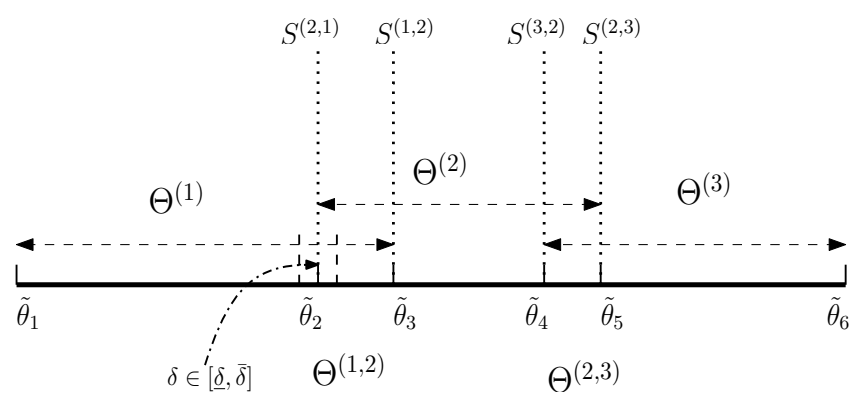

Fig. 1. Illustration of dividing parameter range into multiple subregions with overlap.

where $\mathbf{N}_{J}:=\{1, \cdots, J\}$.

Fig. 1 illustrates the case of 3 subregions with 2 overlapped subsets. Switching surface notation $S^{(i, j)}$ indicates that $j^{\text {th }}$ local LPV controller is activated after $i^{t h}$ local LPV controller. In other words, this means the scheduling parameter moves from subregion $\Theta^{(i)}$ into subregion $\Theta^{(j)}$.

The objective is to design a family of RSSFGS controllers $\mathbf{K}(\tilde{\boldsymbol{\theta}}):=\left\{K_{1}(\tilde{\boldsymbol{\theta}}), K_{2}(\tilde{\boldsymbol{\theta}}), \cdots, K_{J}(\tilde{\boldsymbol{\theta}})\right\}$ of the form,

$$
u(t)=K(\tilde{\theta}) x(t), \quad K \in \mathbf{K}
$$

for each $i-t h$ subregion of scheduling parameters that robustly stabilize the closed-loop system in the presence of noisy scheduling parameters, while minimizing $\mathscr{H}_{\infty}$ norm from disturbance $w(t)$ to the controlled output $z(t)$.

Note that, as the number of subregions increases, less conservative controller can be obtained for each subregion, which leads to improved closed-loop performance. Each controller is designed for the given subregion with the best achievable performance, and the closed-loop system stability is guaranteed over switching surfaces.

The closed-loop system can be obtained by substituting state feedback equation into open-loop system,

$$
\begin{aligned}
& \dot{x}(t)=A_{c l}(\theta(t), \tilde{\theta}(t)) x(t)+B_{w}(\theta(t)) w(t), \\
& z(t)=C_{c l}(\theta(t), \tilde{\theta}(t)) x(t)+D_{w}(\theta(t)) w(t),
\end{aligned}
$$

where

$$
\begin{aligned}
A_{c l}(\theta(t), \tilde{\theta}(t)) & =A(\theta(t))+B_{u}(\theta(t)) K(\tilde{\theta}(t)), \\
C_{c l}(\theta(t), \tilde{\theta}(t)) & =C(\theta(t))+D_{u}(\theta(t)) K(\tilde{\theta}(t))
\end{aligned}
$$

\section{MODELING VARYING PARAMETER}

\section{A. Affine To Multi-simplex Conversion}

Following the procedure presented in [23], [21], the original parameter space needs to be converted into a convex multi-simplex domain. Mult-simplex domain can be defined as the Cartesian product of multiple unit-simplexes. Thus, the actual scheduling parameter $\theta(t)$ and the associated uncertainty $\delta(t)$ can be both converted into unit-simplex variable $\tilde{\alpha}(t)$ as [8],

$$
\begin{array}{ll}
\check{\alpha}_{1}=\frac{\theta(t)+\bar{\theta}}{2 \bar{\theta}}, & \check{\alpha}_{2}=1-\check{\alpha}_{1}=\frac{\bar{\theta}-\theta(t)}{2 \bar{\theta}} . \\
\hat{\alpha}_{1}=\frac{\delta(t)+\bar{\delta}}{2 \bar{\delta}}, & \hat{\alpha}_{2}=1-\hat{\alpha}_{1}=\frac{\bar{\delta}-\delta(t)}{2 \bar{\delta}},
\end{array}
$$


thus, we have $\check{\alpha}=\left(\check{\alpha}_{1}, \check{\alpha}_{2}\right) \in \Lambda_{2}, \hat{\alpha}=\left(\hat{\alpha}_{1}, \hat{\alpha}_{2}\right) \in \Lambda_{2}$. Unitsimplex $\Lambda_{2}$ is defined as:

$$
\Lambda_{2}:=\left\{\alpha \in \mathbf{R}^{2}: \sum_{i=1}^{2} \alpha_{i}=1, \alpha_{i} \geq 0, i=1,2\right\}
$$

Therefore, multi-simplex variable $\tilde{\alpha}=(\check{\alpha}, \hat{\alpha}) \in \Lambda=\Lambda_{2} \times$ $\Lambda_{2}$ can be generated.

Remark 1: All the open-loop system matrices in (1) and controller variables need to be converted into multi-simplex domain using the procedure described above. Note that the open-loop system matrices depend only on the actual scheduling parameters $\theta(t)$. However, the same procedure should be followed to convert them into multi-simplex parameters with the exception that $\bar{\delta}=0$ is substituted in (7). In this case, to distinguish matrices depending on the measured scheduling parameters from system matrices depending on the actual scheduling parameters we denote $\alpha(t)$ for the multi-simplex variables when $\bar{\delta}_{i}=0$. Thus, the system matrices can be written in terms of multi-simplex parameters as, $A(\alpha), B_{w}(\alpha), B_{u}(\alpha), C(\alpha), D_{w}(\alpha)$, and $D_{u}(\alpha)$.

Similarly, varying rate of scheduling parameter and uncertainty can be converted into multiplex variable as:

$$
\frac{\underline{b}_{\theta}}{2 \bar{\theta}} \leq \dot{\tilde{\alpha}}_{1,2} \leq \frac{\bar{b}_{\theta}}{2 \bar{\theta}}, \quad \frac{\underline{b}_{\delta}}{2 \bar{\delta}} \leq \dot{\hat{\alpha}}_{1,2} \leq \frac{\bar{b}_{\delta}}{2 \bar{\delta}}
$$

then,

$$
\Omega_{i}:=\left\{\phi \in \mathbf{R}^{2}: \phi=\sum_{k=1}^{2} \eta_{i k} H_{i}^{k}, \eta_{i} \in \Lambda_{2}, i=1,2\right\}
$$

Therefore, $\dot{\tilde{\alpha}} \in \Omega=\Omega_{1} \times \Omega_{2}$. Note that multisimplex $\Lambda^{i} \times \Omega^{i}$ is used to represent the multisimplex $\Lambda \times \Omega$ for $i=1,2, \ldots, J$ when $\theta$ is restricted to $\Theta^{(i)}$.

A systematic approach and detailed conversion can be found in [8], [23]. The control problem can be expressed in terms of multiplex variables:

Problem 1: Suppose that the scheduling parameter $\theta(t)$ is provided as $\tilde{\theta}(t)$ which is affected by uncertainty $\delta(t)$ (see (2)). For given scalar $\gamma$, find a family of state-feedback switching LPV controllers $K_{j}(\tilde{\theta}(t))$, which stabilizes the closed-loop system (5), and for all parameter pair $(\tilde{\alpha}, \dot{\tilde{\alpha}}) \in$ $\Lambda \times \Omega$, satisfies:

$$
\sup _{\tilde{\alpha}, \tilde{\alpha} \tilde{w}} \sup _{w \in \mathscr{L}_{2}, w \neq 0} \frac{\|z\|_{2}}{\|w\|_{2}}<\gamma
$$

\section{B. Hysteresis Switching by multiple parameter-dependent} Lyapunov functions

Before presenting the synthesis conditions, multiple parameter-dependent Lyapunov function for switching control should be introduced. For each subregion, there is a continuously differentiable parameter-dependent matrix $P_{i}(\alpha)=P_{i}(\alpha)^{\prime}>0$ associated with the $i^{t h}$ subregion. More precisely, the Lyapunov matrix $\left\{P_{i}(\alpha)\right\}_{i \in N_{J}}$ is assumed when the $i^{\text {th }}$ controller is active, then the Lyapunov function can be expressed as,

$$
V_{i}\left(x_{c l}, \alpha\right)=x_{c l}^{T} P_{i}(\alpha) x_{c l}
$$

where, $x_{c l}$ is the closed-loop system state. On the switching surfaces, the condition below should be satisfied,

$$
P_{i}(\alpha) \geq P_{j}(\alpha)
$$

indicating that Lyapunov function of the closed-loop system is non-increasing when switching from $\Theta^{(i)}$ to $\Theta^{(j)}$ that implies

$$
V_{i}\left(x_{c l}, \alpha\right) \geq V_{j}\left(x_{c l}, \alpha\right) .
$$

Then, switching from the $i^{t h}$ controller to the $j^{t h}$ controller is safe.

\section{Gain-Scheduling Controller Synthesis}

Theorem 1: Given a scalar $\gamma$, if there exist contentiously differentiable matrices $0<P_{i}(\alpha)=P_{i}(\alpha)^{\prime} \in \mathbf{R}^{n \times n}, Z_{i}(\tilde{\alpha}) \in$ $\mathbf{R}^{m \times n}, G_{i}(\tilde{\alpha}) \in \mathbf{R}^{n \times n}$, and a scalar $\varepsilon_{i}>0$ for $(\tilde{\alpha}, \dot{\tilde{\alpha}}) \in \Lambda^{i} \times \Omega^{i}$ for $i=1,2, \ldots, J$, such that the following PLMIs are satisfied for $i=1,2, \ldots, J$ :

$$
\left[\begin{array}{cccc}
\Phi_{i, 1}(\tilde{\alpha}, \dot{\tilde{\alpha}}) & * & * & * \\
\Phi_{i, 2}(\alpha, \tilde{\alpha}) & -\varepsilon_{i}\left(G_{i}(\tilde{\alpha})+G_{i}(\tilde{\alpha})^{\prime}\right) & * & * \\
\Phi_{i, 3}(\alpha, \tilde{\alpha}) & \Phi_{i, 4}(\alpha, \tilde{\alpha}) & -I_{p} & * \\
B_{w}(\alpha)^{\prime} & 0_{r \times n} & D_{w}(\alpha)^{\prime} & -\gamma^{2} I_{r}
\end{array}\right]<0
$$

$$
\begin{aligned}
& P_{i}(\alpha) \geq P_{j}(\alpha), \text { if } \alpha \in S^{(i, j)}, \\
& P_{i}(\alpha) \leq P_{j}(\alpha), \text { if } \alpha \in S^{(j, i)},
\end{aligned}
$$

where,

$$
\begin{aligned}
\Phi_{i, 1}(\tilde{\alpha}, \dot{\alpha})= & A(\alpha) G_{i}(\tilde{\alpha})+B_{u}(\alpha) Z_{i}(\tilde{\alpha})+\frac{\partial P_{i}(\alpha)}{\partial \alpha} \dot{\alpha} \\
& +\left(A(\alpha) G_{i}(\tilde{\alpha})+B_{u}(\alpha) Z_{i}(\tilde{\alpha})\right)^{\prime} \\
\Phi_{i, 2}(\alpha, \tilde{\alpha})= & P_{i}(\alpha)-G_{i}(\tilde{\alpha})+\varepsilon_{i}\left(A(\alpha) G_{i}(\tilde{\alpha})+B_{u}(\alpha) Z_{i}(\tilde{\alpha})\right)^{\prime} \\
\Phi_{i, 3}(\alpha, \tilde{\alpha})= & C(\alpha) G_{i}(\tilde{\alpha})+D_{u}(\alpha) Z_{i}(\tilde{\alpha}) \\
\Phi_{i, 4}(\alpha, \tilde{\alpha})= & \varepsilon_{i} C(\alpha) G_{i}(\tilde{\alpha})+\varepsilon_{i} D_{u}(\alpha) Z_{i}(\tilde{\alpha})
\end{aligned}
$$

the gain-scheduling controller with the hysteresis switching logic

$$
K_{i}(\tilde{\alpha})=Z_{i}(\tilde{\alpha}) G_{i}(\tilde{\alpha})^{-1}, \quad i=1,2, \ldots, J
$$

stabilizes the closed-loop system with the guaranteed $\mathscr{H}_{\infty}$ performance bound $\gamma$ for any pair $(\check{\alpha}, \hat{\alpha}) \in \Lambda_{2} \times \Lambda_{2},(\dot{\alpha}, \dot{\alpha}) \in$ $\Omega_{1} \times \Omega_{2}$.

Proof:

This proof can be shown in two portions, stability and robustness on each overlapped subset and stability on switching surfaces.

Inequality (12) can be obtained from Bounded Real Lemma (BRL) of LPV systems with slack variable approach. This inequality implies that the designed controller can stabilize the closed-loop system against scheduling parameter uncertainty in each subset. The detailed proof of this inequality can be found in [8].

On the other hand, inequality (13) guarantees the stability on switching surface by descent Lyapunov matrix on switching surfaces. More precisely, the first inequality in (13) guarantees switching stability when the varying parameter 
$\tilde{\theta}(t)$ (equivalently $\tilde{\alpha}$ ) moves from $\Theta^{(i)}$ to $\Theta^{(j)}$, while the second inequality in (13) guarantees switching stability when the varying parameter moves back from $\Theta^{(j)}$ to $\Theta^{(i)}$.

Based on Theorem 1, the RSSFGS controller design problem is an optimization problem with PLMI constraints and it can be solved by iteratively solving two convex optimization problems with LMI constraints. More specifically, the optimization problem is

$$
\min _{\varepsilon, P(\alpha), Z(\tilde{\alpha}), G(\tilde{\alpha})} \gamma
$$

subject to inequalities (12) and (13).

In practice, the controller is synthesized by optimizing performance for a fixed scalar $\varepsilon$ in terms of time-varying parameters inside specific multi-simplex domain. The value of fixed scalar $\varepsilon$ is chosen by trial and error. However, since the conditions of Theorem 1 are formulated in terms of PLMIs, the optimization problem is of infinite dimensional constraints. Therefore, certain relaxation procedure needs to be applied to convert it into finite dimensional LMIs at the vertices of the multi-simplex domain. In this paper, ROLMIP [22] has been used to perform such manipulations which works jointly with YALMIP [24] and SeDuMi [25].

\section{NUMERICAL EXAMPLE}

Consider this one-dimensional scheduling parameter LPV system [17], [8]:

$$
\begin{gathered}
A(\theta(t))=\left[\begin{array}{cc}
25.9-60 \theta(t) & 1 \\
20-40 \theta(t) & 34-64 \theta(t)
\end{array}\right], \\
B_{u}=\left[\begin{array}{l}
3 \\
2
\end{array}\right], B_{w}=\left[\begin{array}{l}
-0.03 \\
-0.47
\end{array}\right], \\
C=\left[\begin{array}{ll}
1 & 1 \\
0 & 0
\end{array}\right], \\
D_{u}=\left[\begin{array}{l}
0 \\
1
\end{array}\right], D_{w}=\left[\begin{array}{l}
0 \\
0
\end{array}\right] .
\end{gathered}
$$

The scheduling parameter and its varying rate are bounded as $0 \leq \theta \leq 1,|\dot{\theta}(t)| \leq 1$, and measurement noise used in simulations is defined as

$$
\delta(t)=\zeta * \sin (100 * t)
$$

implying that uncertainty and its rate of change are also bounded by $|\delta(t)|<\zeta$ and $|\dot{\delta}(t)| \leq 100 * \zeta$ with $\zeta=0.05$.

For comparison purposes, three different LPV control strategies have been designed for this example. The first one is non-switching LPV controller $(J=1)$. The other two are switching LPV controllers with two $(J=2)$ and three $(J=3)$ subregions, respectively.

More precisely, the non-switching controller is designed to stabilize the system and handle measurement noise of scheduling parameter over the entire range of the parameter space. The range of scheduling parameter variations for this controller is defined as

$$
\Theta:=\{\theta(t) \mid \theta(t) \in[0,1]\},
$$

TABLE I

COMPARISON OF SYSTEM PERFORMANCES OF 3 CASES.

\begin{tabular}{c||c|c|c}
\hline Case & $\gamma_{1}$ & $\gamma_{2}$ & $\gamma_{3}$ \\
\hline \hline non-switching & 0.7777 & - & - \\
\hline Two subregions switching & 0.6980 & 0.6857 & - \\
\hline Three subregions switching & 0.6673 & 0.6582 & 0.4894 \\
\hline
\end{tabular}

For the two-subregion switching case, the parameter set is artificially divided into two overlapped subsets, and two local LPV controllers are designed. Thus, the scheduling parameter range is divided as

$$
\begin{aligned}
& \Theta_{2 s u b}^{(1)}:=\{\theta(t) \mid \theta(t) \in[0,0.6]\}, \\
& \Theta_{2 s u b}^{(2)}:=\{\theta(t) \mid \theta(t) \in[0.4,1]\} .
\end{aligned}
$$

Similarly, for three-subregion switching case, the scheduling parameter set is divided as

$$
\begin{gathered}
\Theta_{3 s u b}^{(1)}:=\{\theta(t) \mid \theta(t) \in[0,0.4]\}, \\
\Theta_{3 s u b}^{(2)}:=\{\theta(t) \mid \theta(t) \in[0.3,0.8]\} . \\
\Theta_{3 \text { sub }}^{(3)}:=\{\theta(t) \mid \theta(t) \in[0.7,1]\} .
\end{gathered}
$$

Obviously, the size of adjacently overlapped subset $\Theta_{j} \cap \Theta_{j+1}$ is larger than that of uncertainty bound, providing enough space for controller to achieve robustness against measurement uncertainty.

Theorem 1 is used to synthesize a family of switching LPV controllers for the overall parameter region. The selection of $\varepsilon_{i}$ is via trial and error, and 0.035 for $\varepsilon_{1}, 0.01$ for all other $\varepsilon$ is chosen to produce a good performance for all three cases.

The system performance of the three designs are compared in Table I. It is clear that as the number of subregions increases, the upper bound of the system performance $\gamma$ decreases. Furthermore, decreasing trend of the performance bound $\gamma$ illustrates that reducing the size of subregions decreases the LPV control synthesis conservativeness and thus leads to improved system performance over the overall parameter region.

Let's assume actual scheduling parameter as linear function of time $t$ in the three subregions, while it is disturbed by sinusoidal noise defined in (17), as shown in Fig. 2.

Additionally, the initial conditions of both LPV plant states are set to $x_{1}(0)=1$ and $x_{2}(0)=-1$, respectively and the disturbance input $w(t)$ is set as a unit pulse with a duration of four seconds. That is, $w(t)=2$ if $t \in[0.5,6]$ and $w(t)$ is zero elsewhere. The LPV plant states are regulated by the designed three LPV controllers (non-switching, switching with two subregions and switching with three subregions).

Fig. 3 and Fig. 4 show the trajectories of system states $x_{1}(t)$ and $x_{2}(t)$ for the non-switching case, two subregions and three subregions switching cases, respectively. Solid curves denote trajectory of states $x_{1}(t)$ or $x_{2}(t)$. Meanwhile, blue color represents non-switching case, red color represents two-subregion case, and black color represents threesubregion case. Black and red dashed lines are switching signal for two-subregion and three-subregion switching cases, 


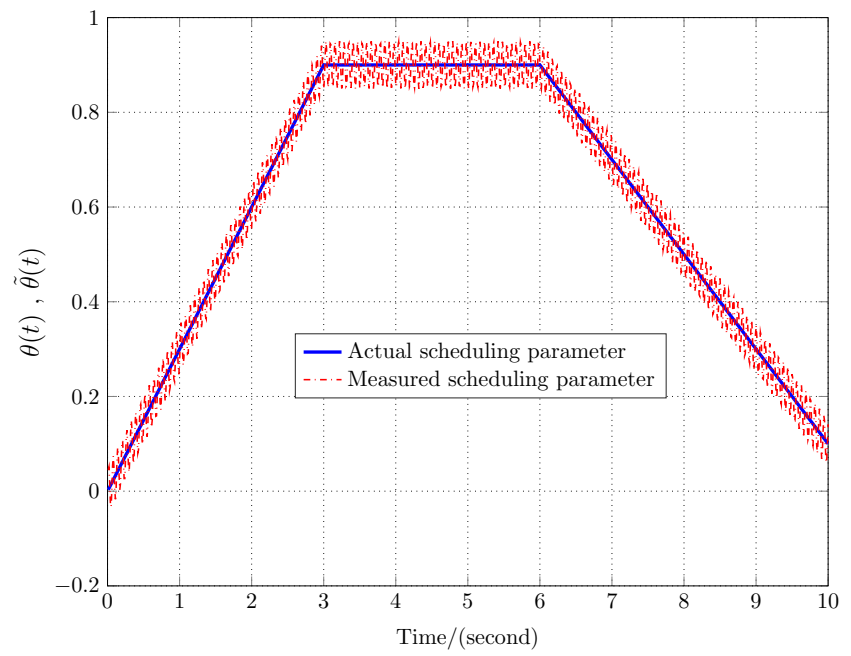

Fig. 2. Trajectory of actual scheduling parameter and measured scheduling parameter.

respectively. Note that, to show switching signal clearly in one figure, signals of two different magnitudes are used to represent the active status of controllers for regions 1 and 2 for the two-subregion case; and signals of 3 magnitudes of indicate the activate status of controllers for regions 1,2 , and 3 for the three-subregion case.

In Figs. 3 and 4, it is easy to observe that when external disturbance $w(t)$ is not applied onto system during $t \in[0,0.5]$ second, the system states are rapidly regulated to zeros. After external disturbance $w(t)$ is applied to the system from $t=0.5$ second, system states are simulated and increases to around 0.3, however, all three controllers (nonswitching LPV, two-subregion and three-subregion RSSFGS controllers) are able to stabilize the closed-loop LPV system. Due to the periodic scheduling parameter uncertainty, controller gains of LPV controllers subsequently fluctuate when disturbance is activated. However, as the number of subregions for the scheduling parameter is increased, the rates of the convergence for both system states become faster, leading to the improved closed-loop system performance. This can also be confirmed by Table I. After external disturbance disappears at $6^{\text {th }}$ second, both system states converge to zero state rapidly. On the moment of switching, i.e. when scheduling parameter is on switching surface, state trajectory goes through smaller overshoot for two-subregion switching case and 3 sub-regions switching case. While, in non-switching case, states $x_{1}(t)$ and $x_{2}(t)$ goes through a large overshoot with the magnitude of 3.0 (outside of the plot in Fig. 3) and 0.8 (Fig. 4). The comparison among three cases clearly demonstrates the advantage of switching LPV controllers in the perspective of system performance.

In Fig. 5, the control signal is plotted for the three different cases. It is clear that the control magnitude is finite but oscillations occur at the switching point (especially near the first second) for the three-subregion case. This is caused by the rapid controller switching on the switching surface and can be solved by adding smooth switching constraints on

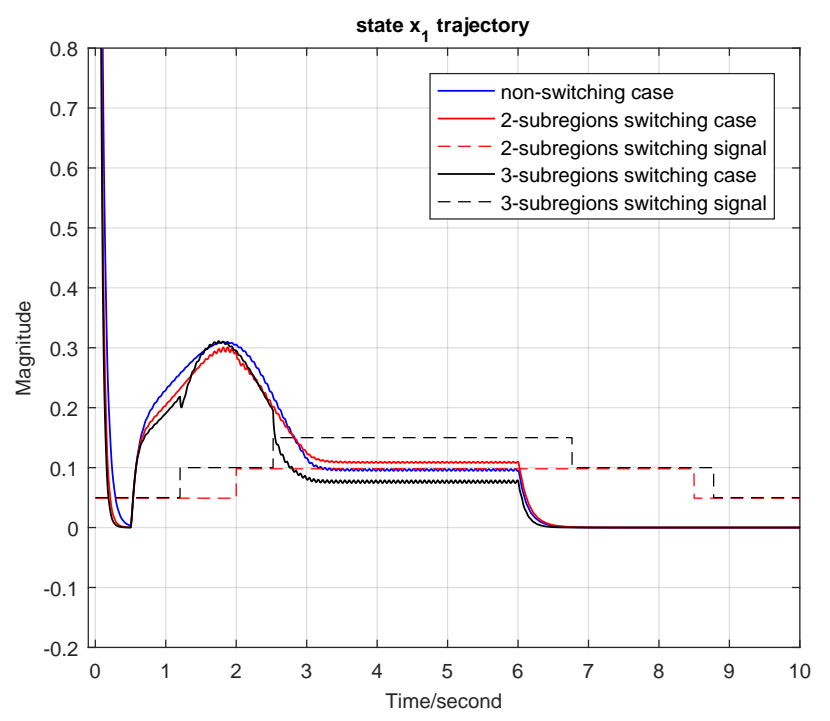

Fig. 3. Response of $x_{1}(t)$ for the three different cases of the control design, where blue, red, and solid lines are for $J=1,2$, and 3; and the red and black dashed lines are for the switching signals (multiplied by 0.05 ) associated with $J=2$ and 3 .

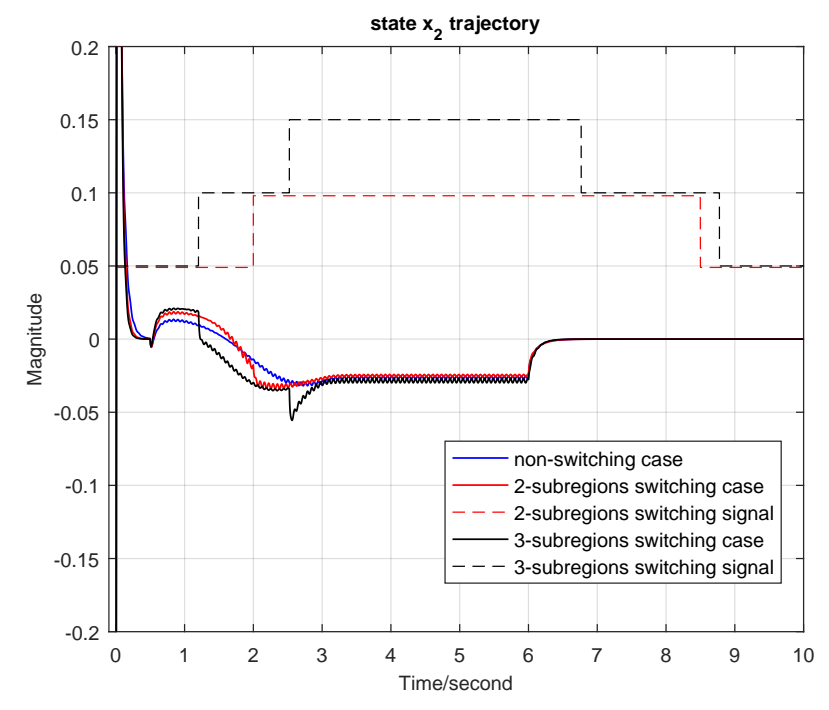

Fig. 4. Response of $x_{2}(t)$ for the three different cases of the control desig, where blue, red, and solid lines are for $J=1,2$, and 3 ; and the red and black dashed lines are for the switching signals (multiplied by 0.05 ) associated with $J=2$ and 3.

PLMIs. This will be the topic for future work.

\section{CONCLUSIONS}

A robust-switching state-feedback (linear parametervarying) LPV control synthesis method is proposed for LPV systems with noisy scheduling parameters. The overall scheduling parameter set is divided into several overlapped subregions. The set of guaranteed performance statefeedback LPV controllers can be designed using the proposed scheme, where hysteresis switching strategy is adopted to robustly switch between adjacent local LPV controllers. 


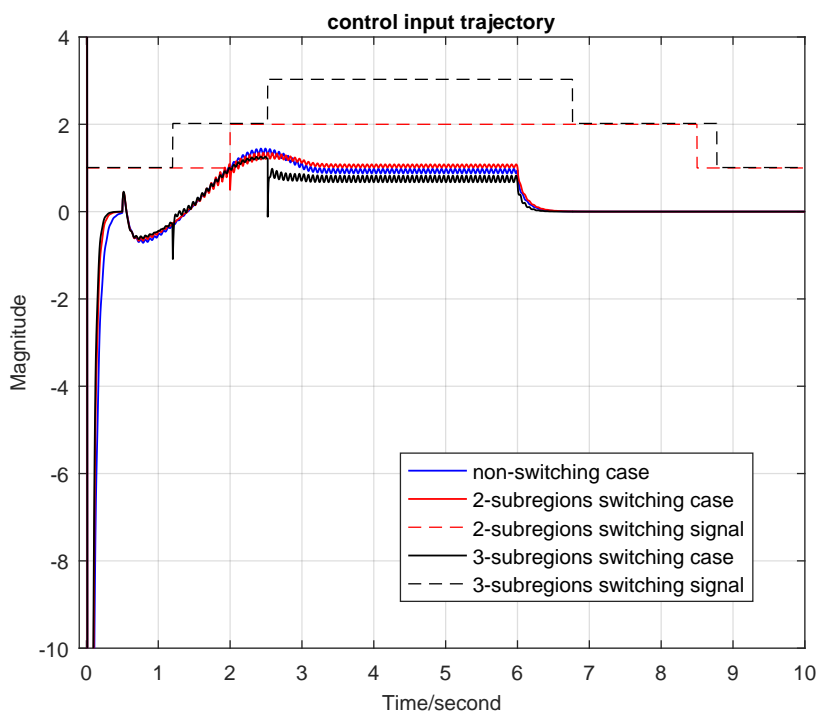

Fig. 5. Control signal associated with the three design cases, where blue, red, and solid lines are for $J=1,2$, and 3; and the red and black dashed lines are for the switching signals associated with $J=2$ and 3.

Parameterized linear matrix inequalities (PLMIs) are formulated based on stability conditions for each subregion and switching stability over each switching surface is guaranteed by multiple parameter-dependent Lyapunov functions. The switching LPV controller is synthesized by solving the feasible solution problem formulated by the derived PLMIs.

A numerical example is given to demonstrate effectiveness of the designed switching LPV controllers. Comparing with non-switching LPV controller, the two-region and threeregion switching LPV controllers reduce the conservativeness of the designed controllers. The future work includes improving the switching smoothness and applying the proposed approach to practical control applications.

\section{REFERENCES}

[1] A. Packard, "Gain scheduling via linear fractional transformations," Systems \& Control Letters, vol. 22, no. 2, pp. 79-92, 1994.

[2] G. Becker and A. Packard, "Robust performance of linear parametrically varying systems using parametrically-dependent linear feedback," Systems \& Control Letters, vol. 23, no. 3, pp. 205-215, 1994.

[3] P. Apkarian, P. Gahinet, and G. Becker, "Self-scheduled $\mathscr{H}_{\infty}$ control of linear parameter-varying systems: a design example," Automatica, vol. 31, no. 9, pp. 1251-1261, 1995.

[4] F. Wu, X. H. Yang, A. Packard, and G. Becker, "Induced $\mathscr{L}_{2^{-}}$ norm control for LPV systems with bounded parameter variation rates," International Journal of Robust and Nonlinear Control, vol. 6, pp. 983-998, Nov. 1996.

[5] W. J. Rugh and J. S. Shamma, "Research on gain scheduling," Automatica, vol. 36, no. 10, pp. 1401-1425, 2000.

[6] A. P. White, G. Zhu, and J. Choi, Linear parameter-varying control for engineering applications. Springer, 2013.

[7] C. Hoffmann and H. Werner, "A survey of linear parameter-varying control applications validated by experiments or high-fidelity simulations," IEEE Transactions on Control Systems Technology, vol. 23, pp. 416-433, Mar. 2015.

[8] A. K. Al-Jiboory, G. G. Zhu, and J. Choi, "Guaranteed performance state-feedback gain-scheduling control with uncertain scheduling parameters," Journal of Dynamic Systems, Measurement, and Control, vol. 138, p. 014502, January 2016.
[9] B. Lu and F. Wu, "Switching LPV control designs using multiple parameter-dependent lyapunov functions," Automatica, vol. 40, no. 11, pp. 1973-1980, 2004.

[10] B. Lu, F. Wu, and S. Kim, "Switching LPV control of an F-16 aircraft via controller state reset," IEEE transactions on control systems technology, vol. 14, no. 2, pp. 267-277, 2006.

[11] J. P. Hespanha, D. Liberzon, and A. S. Morse, "Hysteresis-based switching algorithms for supervisory control of uncertain systems,' Automatica, vol. 39, no. 2, pp. 263-272, 2003.

[12] J. P. Hespanha and A. S. Morse, "Stability of switched systems with average dwell-time," in Decision and Control, 1999. Proceedings of the 38th IEEE Conference on, vol. 3, pp. 2655-2660, IEEE, 1999.

[13] M. Postma and R. Nagamune, "Air-fuel ratio control of spark ignition engines using a switching LPV controller," IEEE Transactions on Control Systems Technology, vol. 20, no. 5, pp. 1175-1187, 2012.

[14] K. Hu and J. Yuan, "On switching $\mathscr{H}_{\infty}$ controllers for nuclear steam generator water level: A multiple parameter-dependent lyapunov functions approach," Annals of Nuclear Energy, vol. 35, no. 10, pp. 1857$1863,2008$.

[15] F. Wu, "Switching LPV control design for magnetic bearing systems," in Proceedings of the 2001 IEEE International Conference on Control Applications, pp. 41-46, IEEE, 2001.

[16] J. Daafouz, J. Bernussou, and J. Geromel, "On inexact LPV control design of continuous time polytopic systems," IEEE Transactions on Automatic Control, vol. 53, pp. 1674-1678, Aug 2008.

[17] M. Sato, Y. Ebihara, and D. Peaucelle, "Gain-scheduled state-feedback controllers using inexactly measured scheduling parameters: $\mathscr{H}_{2}$ and $\mathscr{H}_{\infty}$ problems," in American Control Conference (ACC), 2010, pp. 3094-3099, June 2010

[18] M. Sato and D. Peaucelle, "Gain-scheduled output-feedback controllers using inexact scheduling parameters for continuous-time LPV systems," Automatica, vol. 49, no. 4, pp. 1019-1025, 2013.

[19] A. K. Al-Jiboory and G. G. Zhu, "Improved synthesis conditions for mixed $\mathscr{H}_{2} / \mathscr{H}_{\infty}$ gain-scheduling control subject to uncertain scheduling parameters," International Journal of Control, pp. 1-19, June 2016.

[20] R. Oliveira and P. Peres, "Parameter-dependent LMIs in robust analysis: characterization of homogeneous polynomially parameterdependent solutions via LMI relaxations," IEEE Transactions on Automatic Control, vol. 52, pp. 1334-1340, July 2007.

[21] R. C. L. F. Oliveira, P. Bliman, and P. L. D. Peres, "Robust LMIs with parameters in multi-simplex: existence of solutions and applications," in 47th IEEE Conference on Decision and Control, pp. 2226-2231, Dec 2008

[22] C. M. Agulhari, R. C. L. F. de Oliveira, and P. L. D. Peres, "Robust LMI parser: a computational package to construct LMI conditions for uncertain systems," in XIX Brazilian Conference on Automation (CBA 2012), (Campina Grande, PB, Brazil), pp. 2298-2305, 2012.

[23] M. J. Lacerda, E. S. Tognetti, R. C. Oliveira, and P. L. Peres, "A new approach to handle additive and multiplicative uncertainties in the measurement for $\mathscr{H}_{\infty}$ LPV filtering," International Journal of Systems Science, vol. 47, no. 5, pp. 1042-1053, 2016.

[24] J. Löfberg, " YALMIP : A toolbox for modeling and optimization in MATLAB," in Proceedings of the CACSD Conference, (Taipei, Taiwan), pp. 284-289, September 2004.

[25] J. Sturm, "Using SeDuMi 1.02, a MATLAB toolbox for optimization over symmetric cones," Optimization Methods and Software, vol. 11, no. 1, pp. 625-653, 1999. 